\title{
Cancer Dynamics: Identification of States for Therapeutic Intervention
}

\author{
Amit Jangid ${ }^{1,2}$, Md Zubbair Malik ${ }^{1 *}$, Ram Ramaswamy ${ }^{2 *}$ and R. K. Brojen Singh ${ }^{1 *}$ \\ ${ }^{1}$ School of Computational and Integrative Sciences, Jawaharlal Nehru University, New Delhi 110067, India. \\ ${ }^{2}$ Department of Chemistry, Indian Institute of Technology Delhi, New Delhi 110016, India.
}

\begin{abstract}
We study a minimal model of the stress-driven p53 regulatory network that includes competition between active and mutant forms of the tumor-suppressor gene p53. Depending on the nature of the external stress signal, four distinct dynamical states are observed. These states can be distinguished by different dynamical properties and correspond to active, apoptotic, pre-malignant and cancer states. Transitions between any two of these states are found to be unidirectional and irreversible if the stress signal is either oscillatory or constant. When the signal decays exponentially, the apoptotic state vanishes, and for low stress the pre-malignant state is bounded by two critical points, allowing the system to transition reversibly from the active to the pre-malignant state. For significantly large stress, the range of the pre-malignant state expands and the system moves to the cancerous state which is a stable attractor. This suggests that identification of the pre-malignant state may be important both for therapeutic intervention as well as for drug discovery.
\end{abstract}

Keywords: p53-Mdm2, mutant p53, oncogene, stress, regulatory network, cancer dynamics.

\section{Introduction}

The tumour suppressor gene p53, also termed as the guardian of the genome, crucially determines cell fate through various mechanism [1, 2]. p53 induced different biological outcomes has been studied in detail [4, 5]. Activation of the p53 regulatory pathway by internal and external stress can lead to a number of different outcomes [3, 4]. p53 is known to be mutated in cancer, either in exonic or intronic portion of the gene due to stress [3,6-10]. These mutations eventually lead to disruption in binding DNA. Hence the cell, during transformation, harbours mutated p53 that may finally develop malignancies. From a network theoretical perspective, p53 is a key hub controlling important genes as well as essential cellular functions [1, 11, 12]. Studies on signalling networks have provided identification of many target genes for therapeutic interventions in the context of cancer $[1,11,12]$, although identification of such genes from a dynamical perspective is still open.

Cancer is a complex disease manifested due to the interaction of nonlinear, nonadditive, and dissipative components [13]. In order to understand the functionality of the cell in this state, it is required to know its behavior in the normal state and in perturbed state. Cancer dynamics has been called an emergent property that arises from these interacting components, the constituting genes, small molecules, and the fluctuating environment [14]. p53 holds a central point in signal transduction pathways involving a large number of genes that respond to diverse stress signals [15-18]. This reduces the risk of mutation, and prevents circumstances that can lead to cancer or other pathological states [19]. Since the expression and regulation of p53 depends on its interacting partners in the regulatory pathway, its modeling often involves both negative and positive feedback mechanisms. Mathematical modeling of the p53 regulatory network can provide dynamical information and patterns in order to predict cellular mechanisms and its behavior $[20,21]$ but a challenge is to capture various cellular phases within a simplified minimal model.

p53 is maintained at low levels in the normal condition [22]. Emergence of oscillatory behaviour, one such state in p53 dynamics ("active"), has been extensively studied theoretically and experimentally[27-35]. DNA recovery from low dose of ionizing radiation (IR) corresponds to reversible sustained p53 oscillations with varied amplitude, whereas high dose IR induce irreversible phase leading to stable state (damped oscillations) which corresponds to apoptosis [16-18, 27, 28]. The variability in amplitude of oscillation is found to be larger than the changes in period of oscillation both for damped and undamped conditions [29]. Further, persistent DNA damage activates ATM, and ATM activates Chk2, which results into p53 oscillations to repair damaged DNA[30]. However, what could be the dynamics of p53 in cancer phase is still debatable question.

Some models of p53 capture various possible dynamical states. It is well known that p53 is coupled with Mdm2 via negative feedback loop [27-35]. In these studies it was observed that if negative feedback loop gets activated then DNA repair takes place, whereas, if positive feedback loop gets activated then p53 activation moves to irreversible apoptotic phase. The other regulators of p53 sometimes regulate p53 pulses, for example, inclusion of MDMX in the model system suppress p53 oscillatory amplitude, whereas, knocking out MDMX significantly enhances this amplitude [32]. In recovery phase of DNA damage, the amplitude of the repetitive p53 pulses tends to lower level and become stable normal state (oscillation is minimized or vanished) [33]. However, in the case of apoptosis with excess stress, this amplitude of pulse abruptly rise and moves to irreversible stable state. Similarly, the other regulators of p53 coupled with positive feedback loop (ATM, PTEN, Akt etc) sometimes can induce switching behavior in the p53 dynamical states. Even though these models could able to capture these various dynamical states (active, recovery and apoptosis) which mimic with experimental results in qualitative sense, these models could not able to capture p53 behavior in cancer phase.

Once a normal cell becomes cancerous by mutational process, this signal propagates to neighboring cells [23], thereby a competition is established between normal and cancer cells [24]. The onset, development and propagation of cancer cell population in the normal cell ecology provides a new transformed physico-chemical state, which bears several similarities to first order phase 
transition [25]. A simple "competition" model for cancer is based on two types of cells, normal and cancer with population $N_{1}$ and $N_{2}$. Their dynamics in the cellular ecology can be modeled by the system of equations [26].

$$
\frac{d N_{i}}{d t}=R_{i} N_{i}\left[1-\frac{N_{i}}{K_{i}}-C_{i j} \frac{N_{j}}{K_{i}}\right] \quad \forall i, j=1,2 \mid i \neq j
$$

$R_{i}$, and $K_{i}$ are the intrinsic growth rate and the carrying capacity of species $i$, and $C_{i j}$ is the competition coefficient measuring the effect on species $i$ by species $j$. The equilibrium point (critical point) of the system is $N_{1}=\frac{K_{1}-K_{2} C_{12}}{1-C_{12} C_{21}}$, and $N_{2}=\frac{K_{2}-K_{1} C_{21}}{1-C_{12} C_{21}}$. The normal phase corresponds to the condition $\dot{N}_{1}>0$, and $\dot{N}_{2}<0$ which implies that $C_{12} \leq 0$ and $C_{21} \geq K_{2} / K_{1}$ assuming that $N_{2}<<N_{1}=K_{1}$. Biologically this assumption reflects that in the normal phase, population of cancerous cell is small $(\approx$ 0 ), while population of normal cells reaches its carrying capacity. The condition $\dot{N}_{1}<0$, and $\dot{N}_{2}>0$ which implies $C_{12} \geq 0$ and $C_{21} \leq K_{2} / K_{1}$ corresponds to cancer progression. Apart from normal and cancer phases, there are important dynamical states which may drive the cellular system into various pathologies. Since this dynamical system is function of various species, $\dot{N}_{i}=F_{i}\left(N_{1}, N_{2}, \ldots, N_{m}\right)$; where $i=1 \ldots m$, the exact identification of critical point $\left(\dot{N}_{i}=0\right)$ is difficult.

In this work, we present a minimal p53 regulatory pathway model in order to study phase transition like behavior of normal and cancer in cellular system at molecular level. We also investigate different cancer progression steps captured in the p53 dynamics and possibility of therapeutic intervention in cancer dynamics. We also discussed various key results in the normal to cancer transition in dynamical sense and observations of various stages of cancer phase.

\section{Materials and methods}

\section{Minimal $\mathrm{p} 53_{\mathrm{A}}-\mathrm{p} 53_{\mathrm{M}}-\mathrm{MDM} 2-\mathrm{ARF}$ regulatory network model}

The proposed model is a minimal regulatory network $\mathrm{p} 53_{\mathrm{A}}-\mathrm{p} 53_{\mathrm{M}}-\mathrm{MDM} 2-\mathrm{ARF}$ under stress condition. This involves the interaction of activated p53 $\left(\mathrm{p} 53_{\mathrm{A}}\right)$ and mutated p53 ( $\left.553_{\mathrm{M}}\right)$ along with other key regulators MDM2, ARF and related molecular species as shown in FIG. 1. In the model [35] p53 induces transcription of $\mathrm{RNA}_{\mathrm{N}}$ and is also produced in the nucleus with a constant basal rate. After being produced, it is translated at a constant rate after proceeding in the cytoplasm, and this is followed by eventual decay. Cytoplasmic MDM2 is transported to the nucleus, where it regulates p53 via negative feedback in three different ways. First, transcriptional activity by binding to the p53 transactivation domain [36], second it promotes p53 degradation [37, 38], and finally it favours the export of p53 from the nucleus to cytoplasm [39].

Oncogene activation can be incorporated in the model through either structural alterations (such as chromosomal rearrangement, mutation) or epigenetic modification (gene promoter hypomethylation) [40]. In the present model, we have assumed that a certain type of stress signal $S$ causes structural, and epigenetic modification that result in oncogenes activation. The activated oncogene then activates ARF within the nucleus [41,42] and since ARF is a direct inhibitor of MDM2 activity by binding to the RING finger domain of MDM2 this sequesters MDM2 [43]. Tao, and Levine has observed that ARF blocks the nucleo-cytoplasmic shuttling of MDM2, which is essential for the ability of MDM2 to export p53 into the cytoplasm [44].

Weber and others showed that ARF binds to MDM2 gene and sequesters it into the nucleolus, which in turn prevents p53 regulation by MDM2, and hence leads to the activation of p53 [45]. ARF gets activated due to activation of oncoprotein Myc [45]. It is now well known that activated oncogene, such as c-Myc, leads to the promotion of mutant p53 [46], and this mutated p53 induces the expression of oncogenes [47, 48] as well as inhibits the activity of activated p53 to prevent the cell from apoptosis $[49,49,51-53]$. ARF moves from nucleus to cytoplasm to bind the MDM2 and releases the p53 which is due to activation of oncogene [54].

We incorporate the regulating activity of an oncogene in the p53 network model. In a recent study, it has been shown that regulation/deregulation of c-Myc expression due to stress signal can induce mutation/s in the expression of p53 by binding to $\mathrm{CA}(\mathrm{C} / \mathrm{T})$ GTG-containing site in the p53 promoter [55]. Hence, it has been suggested that stress induced deregulation of c-Myc expression could increase the expression of mutated p53. On the other hand, it has been observed that mutant p53 is able to regulate c-Myc expression by activating c-Myc promoter through C-terminus [47]. Further, it has been reported that p53 repress the c-Myc expression by inducing tumor suppressor miR-145 [56], because c-Myc repression by p53 is required to control the G1 cell cycle arrest [57], such that activation of c-MYC allows the functioning of mutant p53 [58]. Hence, the oncogene we have incorporated in our model is of c-Myc type which allows to interact $\mathrm{p} 53_{\mathrm{M}}$ with oncogene and we studied the dynamical behavior of the model system which gives the similar behavior as the main model (see supplementary information). To keep the model simple we have used either hill function or direct interaction between different proteins, and parameters are estimated to observe the qualitative behavior.

\section{Mathematical framework of the model system}

In the proposed model system (FIG. 1) can be represented by a state vector, $\vec{X}=\left[x_{1}, x_{2}, \ldots, x_{8}\right]^{\top}$, where, $\mathrm{T}$ is the transpose of the vector, and $x_{i} ; i=1,2, \ldots, 8$ represents the concentrations of the corresponding molecular species such that $\vec{X}=\left[p 53_{A}, p 53_{M} \text {, Oncogene, } R N A_{N}, R N A_{C}, M D M 2_{C}, M D M 2_{N}, A R F\right]^{\top}$. Then the model regulatory network is perturbed with a stress with strength $S$ which could be irradiation (IR), molecular (or chemical toxic) fluctuations, environmental fluctuations etc. The amount of stress imparted in the model depends on the $S$ strength, and nature of the $S$ form introduced in the system. In this work, we have taken three different types of nature of stress $S, 1$ ) constant stress form $S=I, 2$ ) periodic stress 


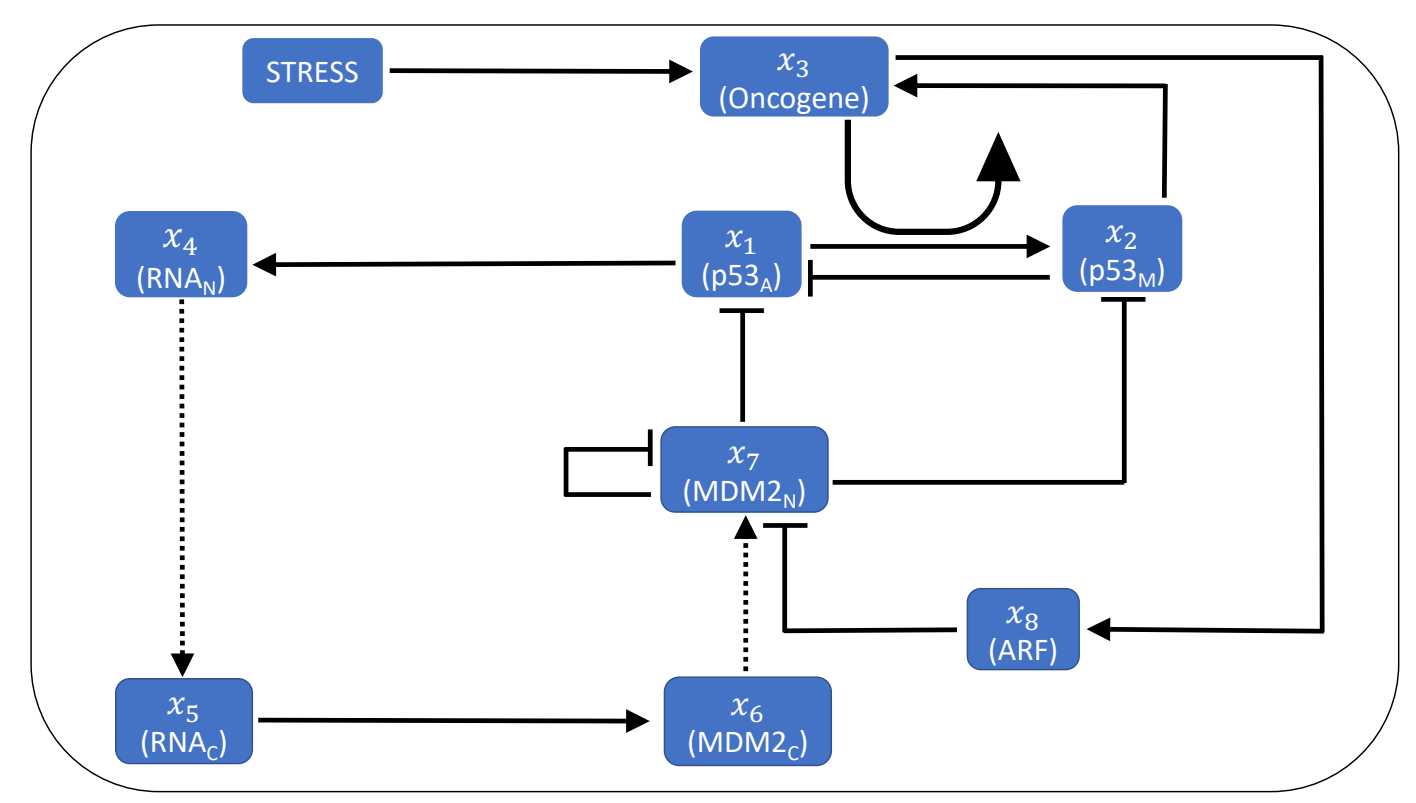

FIG. 1: Interaction network for p53 $3_{A}$-p $53_{M}$-MDM2-ARF-Stress. Dashed arrow shows movement from nucleus to cytoplasm or vice versa, while solid arrow, and bars corresponds to activation, and inhibition on respective node. (Modified network from [35])

$S=I(1+\sin (2 \pi t)) / T,(T=6 \mathrm{hrs}$ through out the model $)$, and 3$)$ exponentially decaying stress $S=I e^{-\lambda t}$. Based on the proposed model system, we arrived at the following set of coupled ordinary differential equations,

$$
\begin{aligned}
\frac{d x_{1}}{d t} & =k_{p}-\left(k_{1} x_{7}+d_{p}+\gamma_{x_{1}} x_{2}+\delta_{x_{1}} \frac{x_{3}^{n_{1}}}{K_{1}^{n_{1}}+x_{3}^{n_{1}}}\right) x_{1} \\
\frac{d x_{2}}{d t} & =\alpha_{x_{2}}+\delta_{x_{1}} \frac{x_{3}^{n_{1}}}{K_{1}^{n_{1}}+x_{3}^{n_{1}}} x_{1}-\gamma_{x_{2}} x_{7} x_{2}-\delta_{x_{2}} x_{2} \\
\frac{d x_{3}}{d t} & =\alpha_{x_{3}}+\beta_{x_{3}} \frac{S^{n_{2}}}{K_{2}^{n_{2}}+S^{n_{2}}}+\delta_{x_{3}} \frac{x_{2} n_{3}}{K_{3}^{n_{3}}+x_{2}^{n_{3}}}-\gamma_{x_{3}} x_{3} \\
\frac{d x_{4}}{d t} & =k_{m}+k_{2} \frac{x_{1}^{1.8}}{k_{D}^{1.8}+x_{1}^{1.8}}-k_{0} x_{4} \\
\frac{d x_{5}}{d t} & =k_{0} x_{4}-d_{r c} x_{5} \\
\frac{d x_{6}}{d t} & =k_{T} x_{5}-k_{i} x_{6} \\
\frac{d x_{7}}{d t} & =k_{i} x_{6}-d_{m n} x_{6}^{2}-k_{3} x_{7} x_{8} \\
\frac{d x_{8}}{d t} & =k_{a}+\delta \frac{x_{3}^{n_{4}}}{K_{4}^{n_{4}}+x_{3}^{n_{4}}} x_{8}-d_{a} x_{8}-k_{3} x_{7} x_{8}
\end{aligned}
$$

Here, $k_{p}$ represents the production rate of $\mathrm{p} 53_{\mathrm{A}}, k_{1}$ is the rate at which $\mathrm{MDM} 2_{\mathrm{N}}$ ubiquitinates $\mathrm{p} 5_{\mathrm{A}}, d_{p}$ is the degradation rate of $\mathrm{MDM} 2_{\mathrm{N}}$ independent of $\mathrm{p} 53_{\mathrm{A}}, \gamma_{x_{1}}$ is the degradation rate due to $\mathrm{p} 53_{\mathrm{M}}$ inhibition. Further, $\delta_{x_{1}}$ shows the rate of mutation in $\mathrm{p} 53_{\mathrm{A}}$ into $\mathrm{p} 53_{\mathrm{M}}$ due to pro-oncogene (oncogenic mutation), $n_{1}$ is Hill coefficient, and $K_{1}$ is the dissociation constant. $\alpha_{x_{2}}$ is the production rate of $\mathrm{p} 53_{\mathrm{M}}$ independent from pro-oncogene (which can be ignored), $\delta_{x_{1}}$ is mutational translation rate of p5 $53_{\mathrm{A}}$ into $\mathrm{p} 53_{\mathrm{M}}$ due to pro-oncogene mutation. Now, $\gamma_{x_{2}}$ is inhibition due to $\mathrm{MDM} 2_{\mathrm{N}}\left(\mathrm{MDM} 2_{\mathrm{N}}\right.$ dependent degradation), and $\delta_{x_{2}}$ shows natural degradation rate of $\mathrm{p} 53_{\mathrm{M}}$ (which can be ignored). $\alpha_{x_{3}}$ is the production rate of pro-oncogene (ONCO) independent of stress (which can be ignored), $\beta_{x_{3}}$ is the stress dependent activation rate of pro-oncogene (oncogene), $n_{2}$ hill coefficient, $K_{2}$ is dissociation constant, $\delta_{x_{3}}$ is the mutated $\mathrm{p} 53_{\mathrm{M}}$ dependent activation rate, $n_{3}$ is Hill coefficient, $K_{3}$ is the dissociation constant, and $\gamma_{x_{3}}$ is the natural degradation rate. $k_{m}$ represents the production rate of nucleic mRNA, $k_{2}$ is the maximum production rate of nucleic mRNA, $K_{D}$ represents dissociation parameter for $\mathrm{p} 53$, and $k_{0}$ is the transportation rate of nucleic mRNA into cytoplasm. $d_{r c}$ represents decay rate of mRNA into cytoplasm. $k_{T}$ represents the translation rate of MDM2 $2_{\mathrm{C}}$, while $k_{i}$ represents nuclear localization of MDM2.$d_{m n}$ is the rate of MDM2 auto ubiquitination, and $k_{3}$ is the degradation rate of MDM2 $2_{\mathrm{N}}$ due to binding ARF to $\mathrm{MDM}_{\mathrm{N}}$. $k_{a}$ is the production rate of ARF, $\delta$ is the maximum activation rate of ARF due to pro-oncogene activation, $n_{4}$ is hill coefficient, $K_{4}$ is the dissociation constant, $d_{a}$ is the natural degradation rate of ARF, and $k_{3}$ is the MDM2 $2_{\mathrm{N}}$ dependent degradation of ARF.

\section{Results}

The system of coupled ordinary differential equations are numerically integrated using ODEINT Python. Numerical simulations are carried out for an arbitrary set of initial values for the variables, and after discarding transients the system dynamics is examined. Initial values of mutant p53, and oncogene are kept zero for each form of the new stress discussed above assuming 

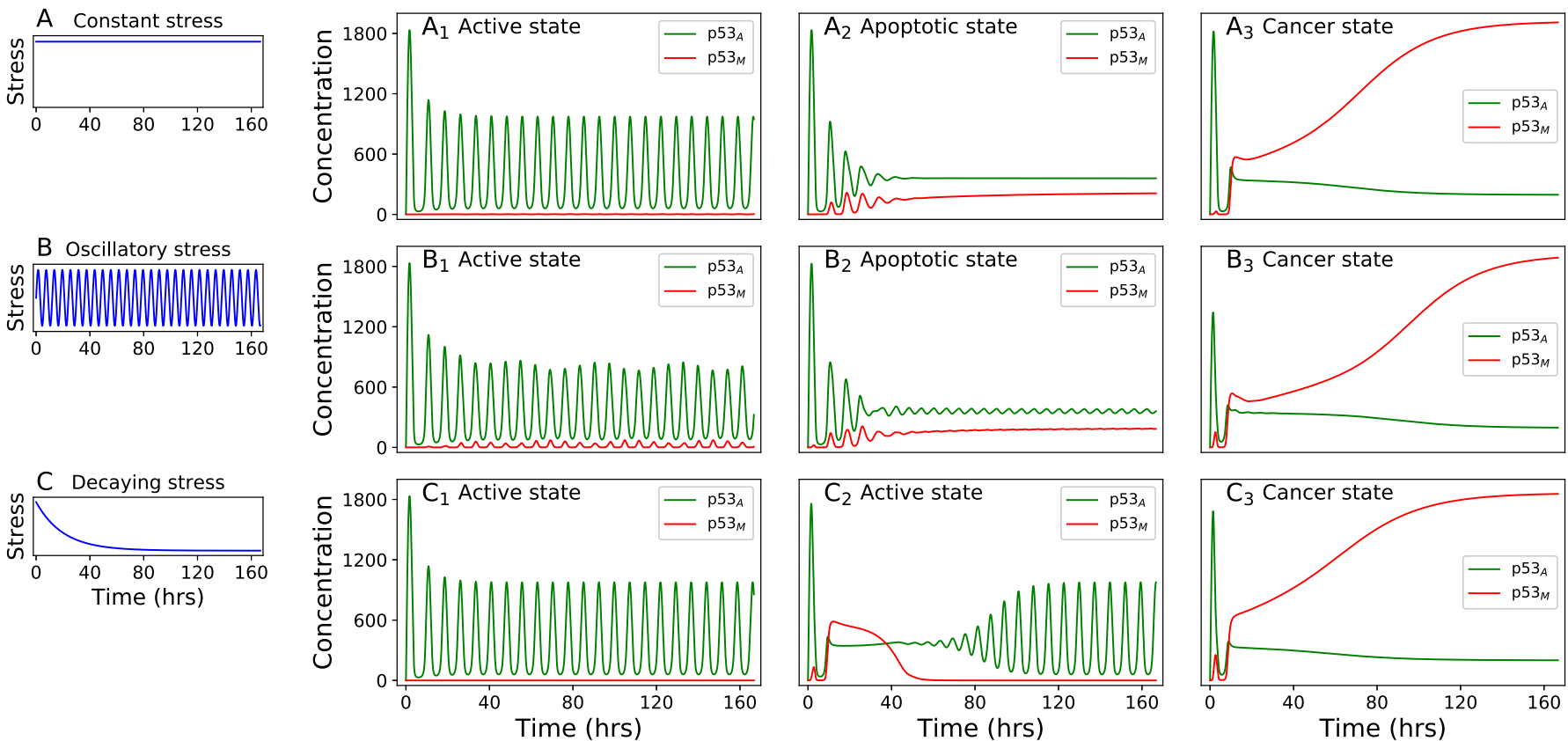

FIG. 2: The left column show three different form of stress discussed about. In the top row $\mathrm{A}_{1}$ (normal), $\mathrm{A}_{2}$ (apoptosis), and $\mathrm{A}_{3}$ (cancer) display the time course of p53 (green), and $\mathrm{p} 53_{\mathrm{M}}$ (red) for constant stress of magnitude $1.00,1.75$, and 2.50 respectively $\left(K_{3}=1000.0\right)$. $\mathrm{B}_{1}$ (normal), $\mathrm{B}_{2}$ (apoptosis), and $\mathrm{B}_{3}$ (cancer) display the time series for averaged oscillatory stress of magnitude 1.00, 1.50 , and 2.50 respectively $\left(K_{3}=1000.0\right)$. And $\mathrm{C}_{1}$ (normal), $\mathrm{C}_{2}$ (recovery from initial cancer stage), and $\mathrm{C}_{3}$ (cancer) display the time series for decaying stress of magnitude $1.00,3.5$, and 4.50 respectively $\left(K_{3}=500.0\right.$ and $\left.\lambda=0.05 \mathrm{hrs}^{-1}\right)$.
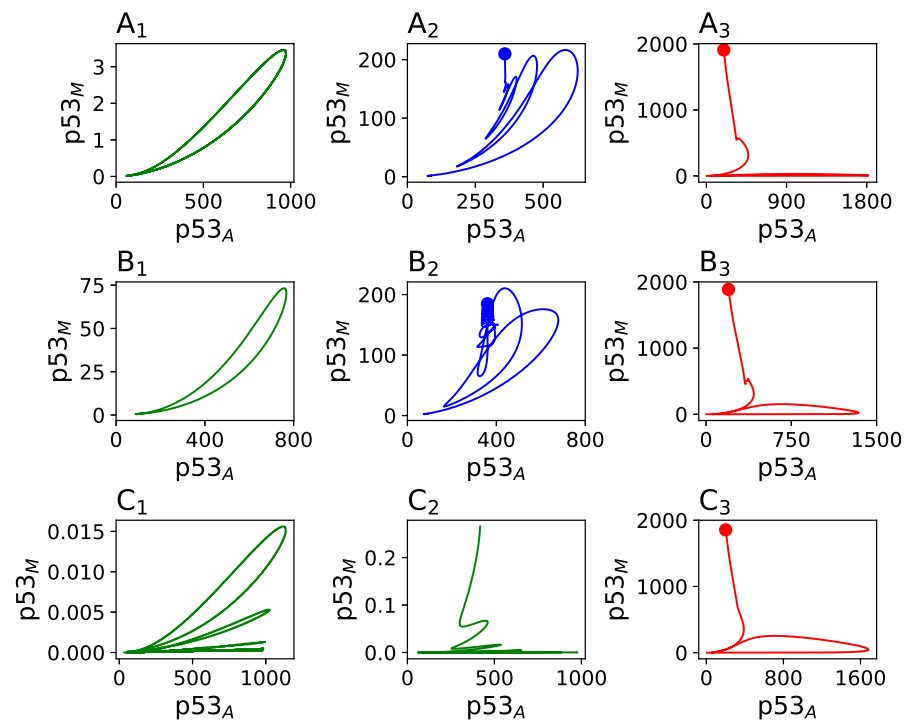

FIG. 3: Dynamics on the phase plane for the time series results in the FIG. 2. Green color indicates active state, blue color apoptotic, and red color cancer state. The dot shows the attractor (end point of the trajectory).

initially there is no mutant $\mathrm{p} 53$, and oncogene.

\section{Phase transition driven by Stress}

Panels $A_{1}, A_{2}$, and $A_{3}$ in FIG. 2 show the time course of $\mathrm{p} 53_{\mathrm{A}}$ and $\mathrm{p} 53_{\mathrm{M}}$ for constant stress signal for three different magnitudes $I=1.0, I=1.75$, and $I=2.5$ respectively. For small magnitude of stress signal $\left(S=1\right.$, FIG. 2 panel $\left.\mathrm{A}_{1}\right)$ both p53 and $\mathrm{p} 53_{\mathrm{M}}$ dynamics show sustained oscillations in which amplitude of oscillations of activated p53 is very high, while the amplitude of mutated p53 is negligibly small. This scenario indicates the possibility of repairing damaged DNA induced by stress signal via $\mathrm{p} 53-\mathrm{MDM} 2$. In such situation, repetitive pulses of $\mathrm{p} 53_{\mathrm{A}}$, which dominate those of $\mathrm{p} 53_{\mathrm{M}}$ in the system, will be generated if damaged DNA is not properly repaired after delivering first pulse. Once the stress is removed, cell comes to the normal state. Hence sustained oscillations of $\mathrm{p} 53_{\mathrm{A}}$ may correspond to the repeated repair efforts of the system to fix damaged DNA.

If the magnitude of the stress signal is significantly high ( $I=1.75$ panel $\mathrm{A}_{2}$ FIG. 2). The system attempts to repair damaged DNA by generating few pulses (five) of activated $\mathrm{p} 53$ (indicated by damped oscillations in $\mathrm{p} 53_{\mathrm{A}}$ and $\mathrm{p} 53_{\mathrm{M}}$ dynamics). This could be the indication that after first pulse, the system sees that the damage is not repairable, it delivers the followed pulses with smaller amplitudes, and moves to amplitude death state [59], $\mathrm{A}_{\mathrm{p} 53_{A}} \rightarrow 0, \mathrm{~A}_{\mathrm{p} 53_{M}} \rightarrow 0$ (when cell dies out due to apoptosis) with 
p $533_{\mathrm{A}}>\mathrm{p} 53_{\mathrm{M}}$. Then p53 pathway activates many apoptogenic genes, by delivering a constant pulse of activated p53, to kill the cell before mutated p53 gets uncontrolled over the p53 $\mathrm{A}$ at stress condition [60, 61]. Alternatively, p53 can also trigger apoptosis by inhibiting antiapoptotic genes (surviving), thus promoting caspase activation [62]. This phase corresponds to apoptotic phase (amplitude death [59] after damped oscillations), where the concentration of $\mathrm{p} 53_{\mathrm{A}}$ still dominates that of $\mathrm{p} 53_{\mathrm{M}}$ in the cellular dynamics.

In the third phase $\mathrm{p} 53_{\mathrm{A}}$ and $\mathrm{p} 53_{\mathrm{M}}$ dynamics, for hight stress $\left(S=2.5\right.$ ), are different from earlier two phases (panel $\mathrm{A}_{3}$ FIG. 2 ). In this phase, $\mathrm{p} 53_{\mathrm{M}}$ concentration grows rapidly, and is high compared to $\mathrm{p} 53_{\mathrm{A}}$ in the normal phase, indicating uncontrolled behavior of $\mathrm{p} 53_{\mathrm{M}}$. This dynamical behavior is qualitatively similar to the experimental observation of higher expression of mutated p53 leads to cancer [63], and in some cancers mutated p53 has dominated effect over active p53 [64]. The normal to cancer transition (NCT) is irreversible: the stress $S$ imparted to the system is able to drive the system into three distinct dynamical states in addition active, apoptosis (indicated by dominant $\mathrm{p} 53_{\mathrm{A}}$, and low $\mathrm{p} 53_{\mathrm{M}}$ ) and cancer (p53 $3_{\mathrm{M}}$ concentration rapidly increasing behavior, and low concentration of $\mathrm{p} 53_{\mathrm{A}}$ with slow decay) states (FIG. 2).

We studied the system dynamics driven by periodic stress of magnitude $I=1.0,1.5,2.5$ (panel $\mathrm{B}_{1}$, $\mathrm{B}_{2}$ and $\mathrm{B}_{3}$ FIG. 2 respectively). We observed three distinct dynamical phases, active, apoptosis, and cancer phase (panels $B_{1}$, $B_{2}$ and $B_{3}$ FIG. 2 respectively), which are qualitatively similar to the constant stress case (panels $\mathrm{A}_{1}, \mathrm{~A}_{2}$ and $\mathrm{A}_{3}$ FIG. 2 respectively). However, the behavior of $\mathrm{p} 53_{\mathrm{A}}$ and $\mathrm{p} 53_{\mathrm{M}}$ in FIG. 2 panel $\mathrm{B}_{2}$ after successive four pulses (with decaying pulses amplitudes), we still observed small amplitude oscillations which do not die out with time which are negligible to the oscillation of active state. Increasing the magnitude, this oscillatory behavior dies out (not shown here). In the case of cancer phase, the monotonically growth of p53 is a little slower as compared to constant stress signal case indicating periodic signal helps the cell to prevent moving to either apoptosis or cancer phase.

The scenario of the behavior of the system dynamics is different in the case of exponentially decay stress. Panel $\mathrm{C}_{1}, \mathrm{C}_{2}$ and $\mathrm{C}_{3}$ FIG. 2 show the time course of $\mathrm{p} 53_{\mathrm{A}}$, and $\mathrm{p} 53_{\mathrm{M}}$ for the magnitude $I=1.0,3.5,4.5$. For $I=1.0$, we observed active state with sustain oscillations (panel $\mathrm{C}_{1}$ FIG. 2). Increasing the stress $(I=3.5)$, the dynamics shows that first, the stress provides a shock to the system allowing $\mathrm{p} 53_{\mathrm{A}}$ moves to amplitude death [59] ( $\mathrm{A}_{\mathrm{p} 53_{A}} \rightarrow$ constant) for small interval of time $T_{p s} \rightarrow[9.8-37]$ hrs, whereas $\mathrm{p} 53_{\mathrm{M}}$ concentration is suddenly increased dominating $\mathrm{p} 53_{\mathrm{A}}$ concentration during $T_{p s}$. Since p $53_{\mathrm{M}}$ dominates over $\mathrm{p} 53_{\mathrm{A}}$ during $T_{p s}$, this state could be considered as a premalignant signature of the system dynamics which can be termed as critical state [25]. During this short time interval $\left(T_{p s} \rightarrow\right.$ finite and $\mathrm{A}_{\mathrm{p} 53_{A}} \rightarrow$ constant), the active state of the system is collapsed, and $\mathrm{p} 53_{\mathrm{M}}$ becomes uncontrollable, and if $T_{p s} \rightarrow \infty$, then the system moves towards cancer phase. Identification of this critical state in cancer patients is very crucial for possible therapeutic intervention for preventing from cancer. After this time interval, the system regains its active state, where, $p 53_{A}$ attains its sustain oscillation state by suppressing p53 $3_{\mathrm{M}}$ concentration level, and then the system repairs damaged DNA. Significantly high dose of the stress signal triggers higher expression of mutated p53 protein than activated p53 which corresponds to the cancer phase. Hence, in case of exponentially decaying stress signal, we are able to observe only two phases active, and cancer phase. Dynamics on the phase plane, for the time series used in the FIG. 2, are shown in FIG. 3. Green color indicates active state (panel $\mathrm{A}_{1}, \mathrm{~B}_{1}, \mathrm{C}_{1}$, and $\mathrm{C}_{2}$ ), blue color apoptotic (panel $\mathrm{A}_{2}$, and $\mathrm{B}_{2}$ ), and red color cancer state (panel $\mathrm{A}_{3}, \mathrm{~B}_{3}$, and $\mathrm{C}_{3}$ ). The dot shows the attractor (end point of the trajectory).

\section{Oncogenic regulation of normal and cancer dynamics}

In this section we study the cooperative impact of oncogene on the dynamics of p5 $3_{\mathrm{A}}$ and p $53_{\mathrm{M}}$ in the regulating pathway. We consider microscopic dissociation parameter $K_{3}$, which is an equilibrium constant that amounts to the probability per unit time to dissociate molecular complex [65]. FIG. 4 shows steady state behavior of $\mathrm{P} 53_{\mathrm{A}}$, and p53 $\mathrm{M}$ as a function of magnitude of stress $(I)$ for three different values of $K_{3}=1000,500,100$. The system's behavior and transition of the states can be studied from steady state behavior (FIG. 4). For oscillatory behavior of p53 $\mathrm{A}$ and $\mathrm{p} 53_{\mathrm{M}}$, the mean population is the average of the maxima and minima of the oscillation calculated in time window of $t=145.82 \mathrm{hr}-166.66 \mathrm{hr}$ (removing transients) while, in case of no oscillations, population of $\mathrm{p} 53_{\mathrm{A}}$, and $\mathrm{p} 53_{\mathrm{M}}$ were taken at time $166.66 \mathrm{hrs}$ (end point of trajectory).

We observed different phases/states (FIG. 4 panels $\mathrm{A}_{1}, \mathrm{~A}_{2}$ and $\mathrm{A}_{3}$ ) in the dynamics of p53 and p53 driven by constant stress for three different values of $K_{3}=1000,500,100$. For small $I$ values $(I\langle 1.19)$, the criteria for this was as average value of $\mathrm{p} 53_{\mathrm{A}}$ reduces by $5 \%$ to its maximum averaged value in the case of without stress, both p $53_{\mathrm{A}}$ and p $53_{\mathrm{M}}$ exhibit oscillatory behavior (FIG. 4 panel $\mathrm{A}_{1}, K_{3}=1000$ ) with the concentration of p53 maintained at minimum level as compared to that of $\mathrm{p} 53_{\mathrm{A}}$. This phase may be considered as active phase of the cellular system, where, p $53_{\mathrm{A}}$ delivers successive pulses to activate various genes which are involved in the pathway to repair damaged DNA. In this case, one can see that difference between $x_{1}$ and $x_{2}$ is almost constant $\left(\Delta x_{12} \rightarrow\right.$ constant $)$. Increasing the strength of the stress $I(I \rightarrow[1.19-2.04])$, we get that $\Delta x_{12}$ becomes variable where, $\mathrm{p} 53_{\mathrm{A}}>\mathrm{p} 53_{\mathrm{M}}$ and $\mathrm{A}_{\mathrm{p} 53_{\mathrm{A}}}, \mathrm{A}_{\mathrm{p} 53_{\mathrm{M}}} \rightarrow$ constant exhibits amplitude death (cell programmed death) scenario in both $\mathrm{p} 53_{\mathrm{A}}$ and $\mathrm{p} 53_{\mathrm{M}}$ dynamics. This state may correspond to apoptotic state (cyan area) in the system dynamics.

In apoptotic phase, the system is not able to repair damaged DNA thereby, p53 $\mathrm{A}$ activates apoptogenic genes favoring to program cell death. It can also be observed that the concentration levels of both p $53_{\mathrm{A}}$ and p $53_{\mathrm{M}}$ are converged to a critical level $x_{c}$ as $I \rightarrow I_{c}=2.04$, which is termed as critical point (FIG. 4 panel $\mathrm{A}_{1}$ ). This critical point can be defined such as: $\lim _{I \rightarrow I_{c}} \Delta x_{12} \rightarrow 0$ and $x_{1}, x_{2} \rightarrow x_{c}$. Slight increase in $I\left(I>I_{c}=2.04\right)$ triggers slow dominance of p53 $\mathrm{M}$ over p53 $\mathrm{A}$, which is the

beginning of new departure to the cancer phase. This new stage can be termed as pre-malignant regime (magenta area). Further increasing $I, \mathrm{p} 53_{\mathrm{M}}$ is found to rapidly increased, while $\mathrm{p} 53_{\mathrm{A}}$ is decreased significantly low, indicating p $53_{\mathrm{A}}$ can no longer control $\mathrm{p} 53_{\mathrm{M}}$ signal such that $\Delta x_{12}$ rapidly increased and then becomes stable. Hence, this phase may be considered as cancer phase (grey area) [25, 66]. In this case, critical point can be seen as the point of departure to either in apoptotic phase or cancer phase.

Decreasing the value of dissociation parameter $K_{3}=500$, we observe similar behavior in p53 $\mathrm{A}$ and p53 dynamics (FIG. 4 panel $\mathrm{A}_{2}$ ), but critical point can be obtained at smaller value of magnitude of stress signal, $I_{c}=1.79$ and range of apoptotic 

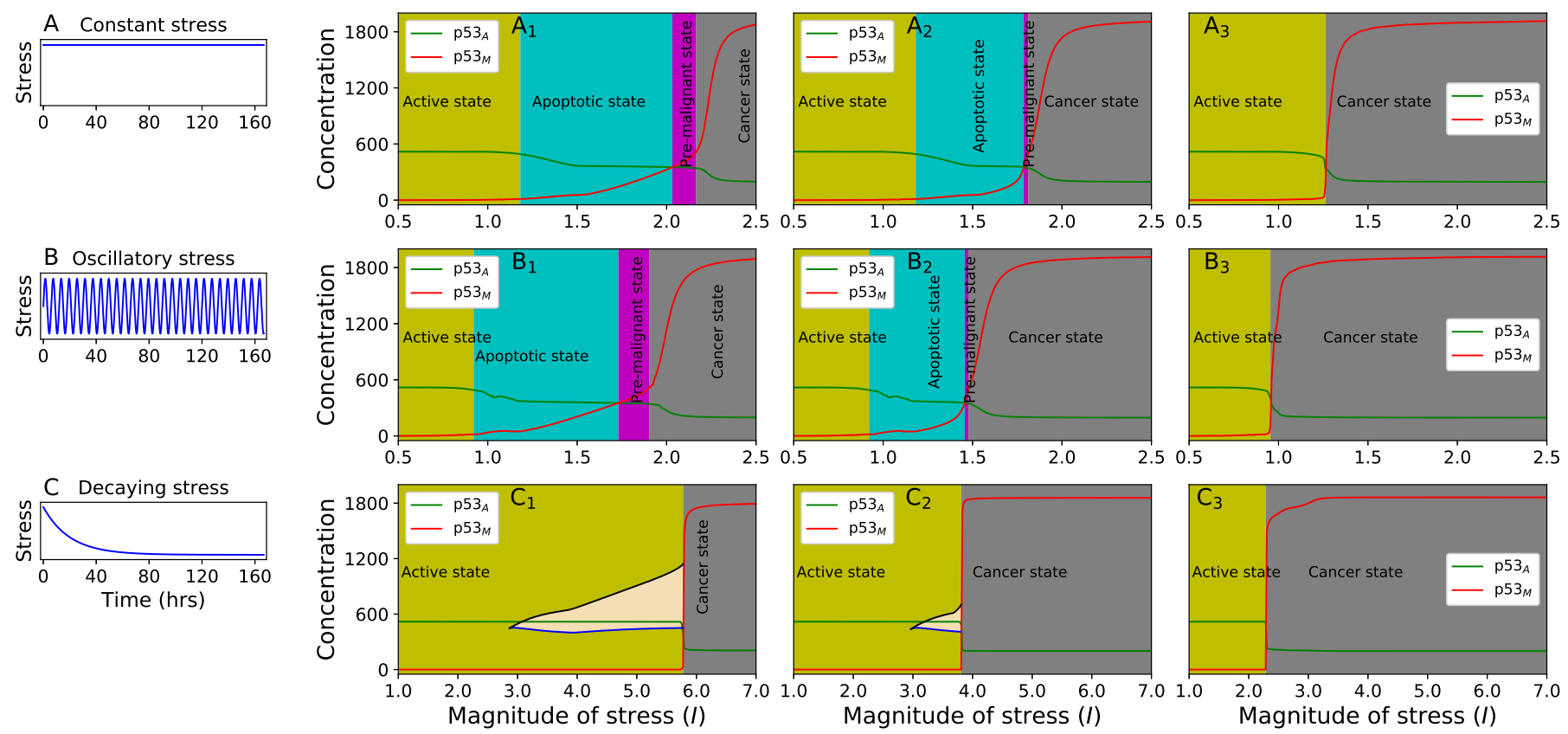

FIG. 4: The left column show three different form of stress discussed about. $A_{1}, A_{2}$, and $A_{3}$ display the steady state behaviour against magnitude of stress for different $\mathrm{K}_{3}$ values 1000.0, 500.0, and 100.0 respectively driven with constant stress. $\mathrm{B}_{1}$, $\mathrm{B}_{2}$, and $\mathrm{B}_{3}$ display the steady state behaviour against magnitude of stress for different $K_{3}$ values 1000.0, 500.0, and 100.0 respectively driven with oscillatory stress. $\mathrm{C}_{1}, \mathrm{C}_{2}$, and $\mathrm{C}_{3}$ display the steady state behaviour against amplitude for different $\mathrm{K}_{3}$ values 1000.0 , 500.0 , and 100.0 respectively driven with decaying stress. Yellow region, cyan region, and grey region correspond to active, apoptotic, premalignant, and cancer state respectively. In panel $\mathrm{C}_{1}$, and $\mathrm{C}_{2}$ (wheat region) black line (upper line), and blue line (lower line) show maximum of p53 $3_{M}$, and maximum of $\mathrm{p} 53_{A}$ in $\mathrm{T}_{p s}$ (see the text) time region, which corresponds to the initial cancer condition.

and pre-malignant state get shrinked and the range of cancer phase increased as compared to the case $K_{3}=1000$ (FIG. 4 panel $\left.\mathrm{A}_{1}\right)$. For comparatively small value of dissociation parameter $K_{3}=100, \Delta I_{m s} \approx 0$ and $\Delta I_{c s} \rightarrow$ large (FIG. 4 panel $\mathrm{A}_{3}$ ). In such situation, a stress state suddenly moves from active to cancer phase crossing critical point without showing the signatures of apoptotic and pre-malignant states, and then become steady $\left(\Delta x_{12} \rightarrow\right.$ constant) both in p53 $\mathrm{A}$ and p53. It may lead to first order phase transition. In case of c-Myc we did not observe pre-malignant regime In constant stress case (supplementary information, panel $\mathrm{A}_{1}, \mathrm{~A}_{2}$, and $\mathrm{A}_{3}$ FIG. 2).

In case of periodic stress, and for same values of $K_{3}=1000,500,100$ (FIG. 4 panels $B_{1}, B_{2}$ and $B_{3}$ ), we observed the similar pattern of four states along with critical point as we found in the case of constant stress. This results also show that all the four states can be obtained at significantly smaller values of stress signal $I$ as compared to those of constant stress case.

We observed different scenario for exponentially decay stress. In this case, we only get three states, active, pre-malignant and cancer state for $K_{3}=1000,500$ and for $K_{3}=100$ we get only two states (FIG. 4 panels $\mathrm{C}_{1}, \mathrm{C}_{2}$ and $\mathrm{C}_{3}$ ). We have also observed that there are two critical points, $x_{c_{1}}$ and $x_{c_{2}}\left(x_{c_{2}}>x_{c_{1}}\right)$ in the range $\Delta I=I_{c_{2}}-I_{c_{1}}$ (wheat region, FIG. 4 panels $\mathrm{C}_{1}$ and $\mathrm{C}_{2}$ ). In this range $\Delta I, \mathrm{p} 53_{\mathrm{M}}$ dominates over $\mathrm{p} 53_{\mathrm{A}}$ for a certain time interval $T_{p s}$ (previous section), which is a signature of pre-malignant or critical state, which comes back to the active state after $T_{p s}$ time interval if $I \in\left[I_{c_{1}}, I_{c_{2}}\right]$, where, $I_{c_{2}}=5.80$ for $K_{3}=1000$ and $I_{c_{2}}=3.83$ for $K_{3}=500$. In the dynamical system study, the identification of this critical point/s and pre-malignant regime of any cancer type are quite important for therapeutic intervention of the cancer [25]. The reason could be if system dynamics is in this regime $I \in\left[I_{c_{1}}, I_{c_{2}}\right]$, there is a possibility of repairing damaged DNA. For lower value of $K_{3}$ parameter $\left(K_{3}=100\right)$, if $I>I_{c_{2}}$ the two critical points become single $I_{c_{1}}=I_{c_{2}}>I_{c}$, and the active state directly jumps to cancer state $\left(T_{p s} \rightarrow \infty\right)$ via $I_{c}$ (FIG. 4 panels $\mathrm{C}_{3}$ ). These critical points can be seen as the points of departure to either in active state or cancer state. All these results indicate that the impact of oncogene is quite significant in regulating normal and cancer dynamics as well as their state transition.

\section{Phase transition and key to therapeutic intervention}

In this section we study the dynamical behavior of $\mathrm{p} 53_{\mathrm{A}}$, and $\mathrm{p} 53_{\mathrm{M}}$ in two parameter space driven by different stress (FIG. 5 panel A). Each point in two parameter space (Magnitude of stress, $K_{3}$ ) (FIG. 5 panel A) are calculated concentrations of $p 53_{A}$ in the dynamics: for oscillatory dynamics each point is the average of maxima and minima obtained in the time interval [145.82, 166.66] hrs, otherwise (no oscillation) concentration are measured at time 166.66 hrs. FIG. 5 A (with constant stress) shows three distinct regimes/phases active (green region), apoptosis (yellow region) and cancer (red region). For large value of $K_{3}$, transition from active to cancer state is through apoptotic phase, while for low value of $K_{3}$, the range of apoptotic regime is so thin that slight increase in stress magnitude $(I)$ might lead to direct cancer phase. Transition from active to apoptotic state is one directional. Figure $\mathrm{A}_{1}, \mathrm{~A}_{2}, \mathrm{~A}_{3}$, and $\mathrm{A}_{4}$ are the time course at different point on the heat map (FIG. 5 A).

Similar behavior was observed in the patterns of two parametre space in case of periodic stress (FIG. 5 panel B). The panels $\mathrm{B}_{1}, \mathrm{~B}_{2}, \mathrm{~B}_{3}$, and $\mathrm{B}_{4}$ show the corresponding time series for the parameter set $(0.5,500.0),(1.3,500.0)$, (0.96, 100.0), and $(2.0$, 200.0) respectively on the heat map. It is also observed that in case of periodic stress, less magnitude of stress is required for 

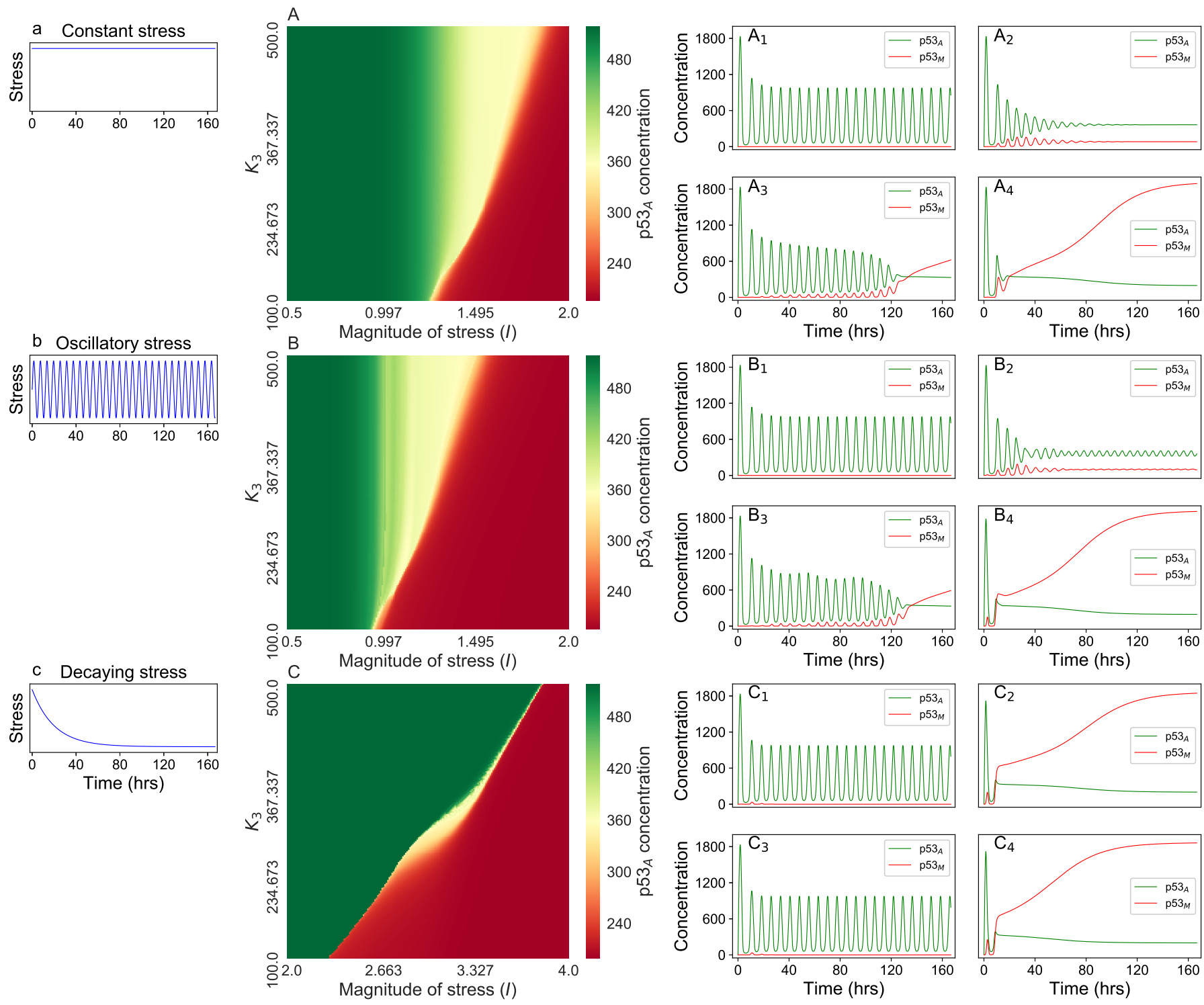

FIG. 5: The left column show three different form of stress discussed about. Second column (A, B, and C) show two parameter steady state behavior of the system for three different form of driven stress ( $\mathrm{a}$, b, and c) respectively. $\mathrm{A}_{1}, \mathrm{~A}_{2}, \mathrm{~A}_{3}$, and $\mathrm{A}_{4}$ correspond to the time course of $\mathrm{p} 53_{\mathrm{A}}$ (green), and $\mathrm{p} 53_{\mathrm{M}}$ (red) for the parameter set $(0.5,500.0),(1.6,500.0),(1.27,100.0)$, and $(2.0,200.0)$ respectively on the heat map A. $\mathrm{B}_{1}, \mathrm{~B}_{2}, \mathrm{~B}_{3}$, and $\mathrm{B}_{4}$ correspond to the time course for the parameter set $(0.5,500.0),(1.3,500.0),(0.96,100.0)$, and $(2.0,200.0)$ respectively on the heat map B. And $\mathrm{C}_{1}, \mathrm{C}_{2}, \mathrm{C}_{3}$, and $\mathrm{C}_{4}$ for the parameter set $(2.0,500.0),(4.0,500.0),(2.0,100.0)$, and (4.0, 100.0) respectively on the heat map C. First, and second term in the parameter set correspond to magnitude of stress $(I)$, and $K_{3}$ respectively. Green, yellow, and red region indicate active, apoptotic, and cancer phase respectively on the heap map (A, B, and C).

different phase transition than constant stress.

In case of exponentially decay stress, we observed only two states active (green region), and cancer (red region) (FIG. 5 panels C) unlike constant, and periodic stress. The significantly small yellow region as compared to active and cancer regions is observed in the phase diagram indicating either active state or cancer state (due to $166.66 \mathrm{hrs}$ window). Further, the behavior also suggests that increasing the magnitude of stress signal, and decreasing $K_{3}$ parameter value enhances the chance of inducing cancer phase in the system dynamics. Hence, $K_{3}$ parameter is a crucial parameter for cancer dynamics where low value of $K_{3}$ leads to more chances of having cancer [66].

The results discussed above indicate that apart from different stress, introduced in the system, there are various other factors which can drive the system to cancer state, for example, oncogene and its associated pathway/s. These factors are in fact the key to sustain the system at active state or bring back to active state from pre-malignant state by regulating these parameters and their associated pathways. Moreover, the identification of these critical point/s and pre-malignant state is very important.

\section{Cancer recovery phase: dynamics of pre-malignant state}

In this section we focus on the properties of the pre-malignant, and critical point/s, and their importance in therapeutic intervention to prevent the cancer. As we have discussed in previous sections, we could able to find only one critical point $\left(T_{c}\right)$ for constant, and periodic stress driven system (FIG. 2, 4 and 5). In these cases the pre-malignant state is just the beginning of cancer state, and it is hard to bring back to normal state. The scenario is quite different for exponentially decay stress. Here, we study the recovery time behavior for three different set of paramaters such as (magnitude of stress, and $K_{3}$ ), (magnitude of stress, and $K_{4}$ ), and (magnitude of stress, and $\lambda$ ) (FIG. 6). In this case, we observed two critical points $T_{c_{1}}>0$ and $T_{c_{2}}>0$ 

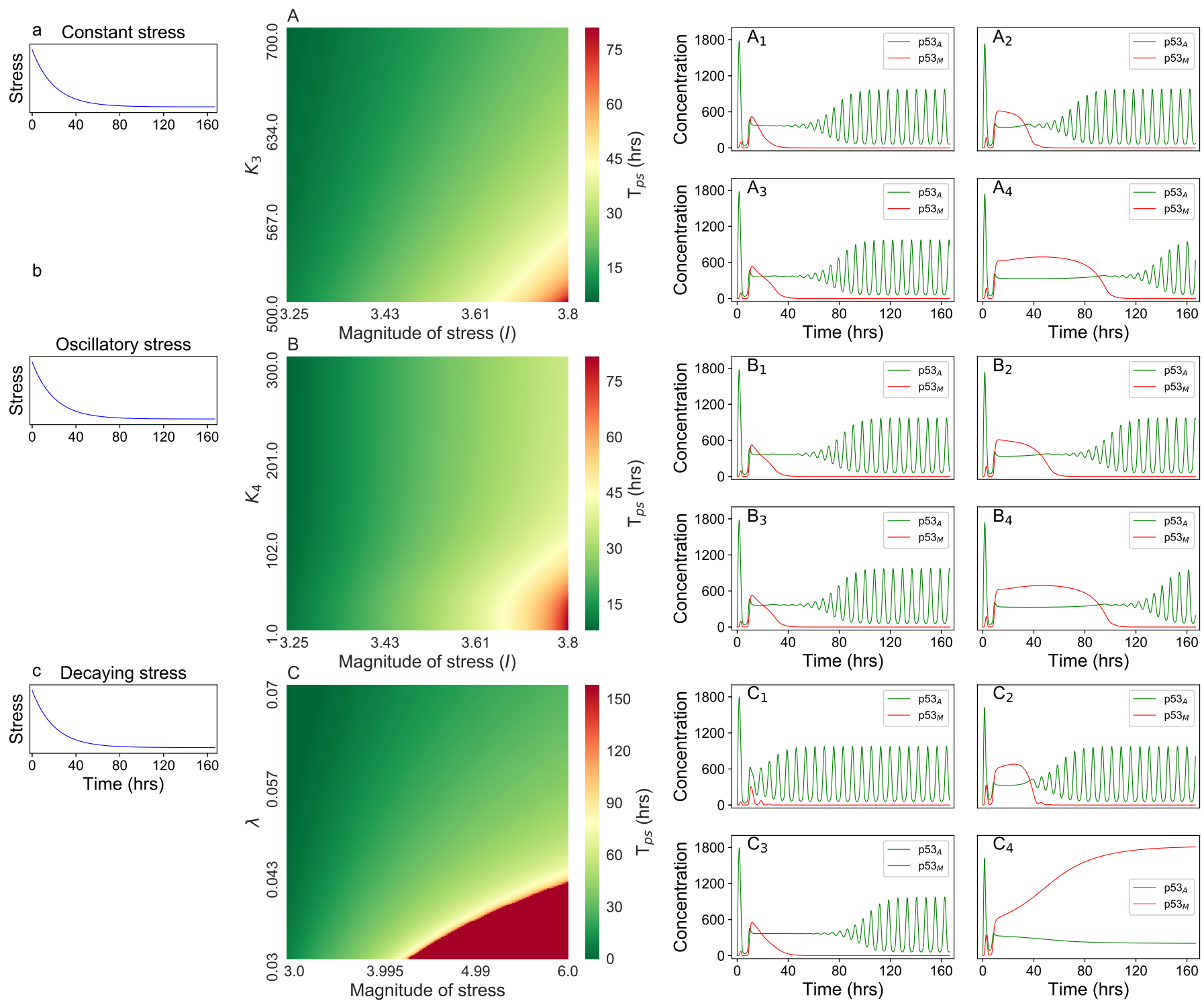

FIG. 6: The left column show decaying stress. Second column, A, B, and C, show two parameter cancer recovery behavior for the parameter set (magnitude of stress, $K_{3}$ ), (magnitude of stress, $K_{4}$ ), and (magnitude of stress, $\lambda$ ) respectively driven with same decaying stress. $\mathrm{A}_{1}, \mathrm{~A}_{2}, \mathrm{~A}_{3}$, and $\mathrm{A}_{4}$ correspond to the time course for the parameter set $(3.25,700.0),(3.8,700.0)$, (3.25, 500.0), and (3.8, 500.0) respectively on the heat map $\mathrm{A} . \mathrm{B}_{1}, \mathrm{~B}_{2}, \mathrm{~B}_{3}$, and $\mathrm{B}_{4}$ correspond to the time course for the parameter set $(3.25,300.0),(3.8,300.0),(3.25,1.0)$, and $(3.8$, 1.0) respectively on the heat map $\mathrm{B}\left(K_{3}=500.0\right) . \mathrm{C}_{1}, \mathrm{C}_{2}, \mathrm{C}_{3}$, and $\mathrm{C}_{4}$ correspond to the time course for the parameter set $(3.0,0.07)$, $(6.0,0.07),(3.0,0.03)$, and $(6.0,0.03)$ respectively on the heat map $\mathrm{C}\left(K_{3}=1000.0, K_{4}=10.0\right)$. On the heat map green color shoes lowest recovery time, while red shows highest recovery time or no recovery (in case of cancer)

with $T_{c_{2}}>T_{c_{1}}$ separated by a time interval $T_{p s}=T_{c_{2}}-T_{c_{1}} \geq 0$ in the p53 $3_{\mathrm{A}}$ and p $53_{\mathrm{M}}$ dynamics. However, for time $<T_{c_{1}}$ and time $>T_{c_{2}}$, the system dynamics will be in active state, where $\mathrm{p} 53_{\mathrm{A}}$ dynamics showed sustain oscillatory behavior controlling $\mathrm{p} 53_{\mathrm{M}}$ dynamics to maintain at minimum concentration level $\left(\mathrm{p} 53_{\mathrm{M}}<\mathrm{p} 53_{\mathrm{A}}\right)$. This particular state is termed as pre-malignant state (discussed earlier), and is shown in FIG. 6. For certain values of the parameter set we observed that the system dynamics show a situation, $T_{p s} \rightarrow \infty, T_{c_{1}} \rightarrow T_{c_{2}} \rightarrow T_{c}$ and $\mathrm{p} 53_{\mathrm{M}}>\mathrm{p} 53_{\mathrm{A}}$ exhibit stable attractor, then the dynamical system becomes cancer state. In this case, we did not observed apoptotic state.

We observe that by decreasing $K_{3}$ and increasing the magnitude of stress $I, T_{p s}$ is increased, but $T_{c_{1}} \rightarrow \operatorname{constant}($ same), which is pre-malignant state (FIG. 6 panels $A_{1}-A_{4}$ ) in the parameter space of $I$, and $K_{3}$. In such situation, there is always a possibility of bringing back into active state. However, for significantly small $K_{3} \leq K_{3}^{c}$ and large $I \geq I^{c}$, where, $K_{3}^{c}$ and $I^{c}$ being critical values, we could able to observe the cancer state condition: $\lim _{\left(K_{3} \leq K_{3}^{c}, I \geq I^{c}\right)} T_{p s} \rightarrow \infty, T_{c_{1}} \rightarrow T_{c_{2}} \rightarrow T_{c}$ and $p 53_{M}>p 53_{A}$ exhibiting stable attractor. Once the system reaches this phase, the dynamical process of the system becomes irreversible, and the system could not back to active state. Similar behavior and dynamical patterns can be found for set of $(I$, and $\left.K_{4}\right)$ in FIG. 6 panel B and $B_{1}-B_{4}$, and for set of $(I, \lambda)$ in FIG. 6 panels C, $C_{1}-C_{4}$, where we could see the three states distinctly.

From the perspective of dynamical system analysis, identification of these three states obtained in any type of cancer is quite important in view of prevention from that cancer. The reason could be due to the possibility of bringing back to normal condition from pre-malignant signature. Proper therapeutic intervention and drug administration needed to be done during the time $T_{p s}$ to prevent from cancer phase. It may not be able to cure the cancer once proper intervention and preventive measures are not taken up. Further, for the sake of cancer drug discovery, this pre-malignant state could be proper stage of investigation. 


\section{Discussion and Summary}

A dynamical systems approach can offer fresh insights to understanding cancer progression, and therefore suggest new protocols in therapeutic intervention. Cancer can be treated in broadly in two ways by exploring dynamical behavior along with hidden patterns of cancer and associated cellular states, and second to explore proper cellular state and time for therapeutic intervention or drug discovery. In the present work we have studied a model that incorporates the dynamics of both active and mutant p53 that are driven by different forms of time-dependent stress, and have considered the impact of ARF and oncogenes through different feedback mechanisms. This simple model has four distinct final states that can be characterised by the asymptotic dynamics: these have experimental validation $[29,67]$ and variously correspond to active, apoptotic, pre-malignant and cancer states.

Sustained oscillations in $\mathrm{p} 53_{A}$ and $\mathrm{p} 53_{M}$ dynamics can be seen as repeated pulses that occur in the system when DNA damage is repaired. Such oscillations persist until the DNA repair is completed [33]. Stress that triggers the system to the active state is a reversible process, the dynamics reverting to normal when the stress is removed. For high stress or when there are $p 53_{M}$ activators such as oncogene and/or ARF, the amplitude of $\mathrm{p} 53_{A}$ oscillation with be large enough to arrest the cell cycle. In this situation, the amplitude of $p 53_{M}$ reaches a critical level, although lower than the amplitude of $\mathrm{p} 53_{A}$ [68]. Oscillatory dynamics vanishes [59] for both $\mathrm{p} 53_{A}$ and $\mathrm{p} 53_{M}$; this is a state of amplitude death leading to a stable fixed-point attractor. This corresponds to apoptosis since the system cannot revert to oscillatory dynamics: this is an irreversible transition [25].

For large stress the production of mutant $\mathrm{p} 53_{M}$ becomes rapid and uncontrolled. The concentration level of p53 $3_{M}$ exceeds a critical apoptotic threshold, and this can be seen as a stress-induced premature senescence. This suppresses apoptosis and triggers cancer progression [69]. For constant or a periodic stress signal, we were able to find a condition where p53 $3_{A}$ and p $53_{M}$ coincide. We term this a critical point of the dynamical system, and this can be considered as leading to a new, cancer, state: mutant $\mathrm{p} 53_{M}$ is uncontrollable $\left.\left(p 53_{M}\right\rangle p 53_{A}\right)$. Furthermore, there is no possibility of DNA repair and the process is irreversible. However, there is a small range of stress where the concentration of mutant p53 increases slowly, compared to the monotonic increase in the cancer regime. This we term pre-malignant. For constant or periodic stress there is a single critical point and hence the system, having transitioned to the cancer state cannot revert to the normal state.

For exponentially decaying stress only three states can be observed: active, pre-malignant or cancer. There are two critical points in this case, indicating the possibility of reversing from the pre-malignant to the active state. The width of the transition region depends on the stress inducing parameters with respect to oncogene, ARF, and other mechanisms. Identification of this range of the pre-malignant state, along with critical points, is important for therapeutic intervention.

Our study provides a qualitative picture of the dynamical properties of states observed in various experiments on cellular dynamics. The present results indicate the possibility of measuring how much stress suffices to lead to cancer. It will be important to explore the role of noise in driving the dynamics to see how robust these results are to extrinsic or intrinsic stochasticity.

\section{Acknowledgments}

AJ (21/06/2015(i)EU-V) is financially supported by University Grants Commission of India, New Delhi, India. MZM is financially supported by Department of Health and Research, Ministry of Health and Family Welfare, Government of India (File No. R.12014/01/2018-HR, FTS No. 3146887). RR is financially supported by JC Bose Fellowship, Department of Science and Technology, India. RKBS acknowledges UPE-II (101) for providing financial support.

\section{Author Contributions}

AJ and MZM conceived the model. AJ, MZM, RR and RKBS did analytical work. AJ, MZM, RR and RKBS did the numerical experiment and prepared the figures of the numerical results. AJ, MZM, RR and RKBS analyzed and interpreted the analytical as well as simulation results. All authors wrote and approve the final manuscript.

\section{Additional Information}

Additional information is available at

\section{Conflict of Interest Statement}

None declared.

\section{Model simulation}

Numerical integration were carried out using Python ODEINT. 
[1] D. P. Lane. p53, guardian of the genome. Nature, 358:15-16, 1992.

[2] H. V. Karen. p53: Death star. Cell, 103(5):691 - 694, 2000.

[3] T. B. Mark and V. Nikolina. p53: a molecular marker for the detection of cancer. Expert Opinion on Medical Diagnostics, 2(9):1013-1024, 2008. PMID: 23495923.

[4] N. Issaeva. p53 Signaling in Cancers. Cancers, 11(3), 332, 2019. doi:10.3390/cancers11030332.

[5] K.H. Vousden, and X. Lu. Live or let die: the cell's response to p53. Nat. Rev. Cancer, 2:594-604, 2002.

[6] A. J. Levine. The many faces of p53: something for everyone. J Mol Cell Biol, 11(7):524-530, 2019. doi: 10.1093/jmcb/mjz026.

[7] D. W. Meek. Regulation of the p53 response and its relationship to cancer1. Biochemical Journal, 469(3):325-346, 07 2015.

[8] A. V. Gudkov and E. A. Komarova. The role of p53 in determining sensitivity to radiotherapy. Nature Reviews Cancer, 3(2):117-129, 2003.

[9] M. Oren. Decision making by p53: life, death and cancer. Cell Death Dierentiation, 10(4):431-442, 2003.

[10] T. Soussi. The p53 pathway and human cancer. BJS, 92(11):1331-1332, 2005.

[11] K. Hiroaki. Foundations of Systems Biology. 2001.

[12] B. Alberts, A. Johnson, J. Lewis, M. Ra, K. Roberts, P. Walter, and N. Chaey. Molecular biology of the cell. 4th edn. Annals of Botany, 91(3):401-401, 1991.

[13] L. Reinhard, H. Valerie, J. Abdul, V. T. Suzy, S. Vladimir, M. Pedro, M. T. Frank, and A. Steven. A systems biology view of cancer. Biochimica et Biophysica Acta (BBA) - Reviews on Cancer, 1796(2):129 - 139, 2009.

[14] E. E. Schadt. Molecular networks as sensors and drivers of common human diseases. Annals of Botany, 461:218-223, 2009.

[15] C. J. Proctor and D. A. Gray. Explaining oscillations and variability in the p53-mdm2 system. BMC Systems Biology, 2:75, 2008.

[16] M. Z. Malik, S. Ali, S. S. Singh, R. Ishrat, and R. K. B. Singh. Dynamical states, possibilities and propagation of stress signal. Scientic Reports, 7:40596, 2017.

[17] M. Z. Malik, S Ali, M. J. Alam, R. Ishrat, and R. K. B. Singh. Dynamics of p53 and wnt cross talk. Computational Biology and Chemistry, 59:55 - 66, 2015. Advances in Systems Biology.

[18] M. Z. Malik, M. J. Alam, R. Ishrat, S. M. Agarwal, and R. K. B. Singh. Control of apoptosis by smar1. Mol. BioSyst., 13:350-362, 2017.

[19] A. J. Levine, W. Hu, and Z. Feng. The p53 pathway: what questions remain to be explored . Cell Death Dierentiation, 13:1027-1036, 2006.

[20] H. M. Byrne. Dissecting cancer through mathematics: from the cell to the animal model. Nature Reviews Cancer, 10:221-230, 2010.

[21] P. M. Altrock, L. L. Liu, and F. Michor. The mathematics of cancer: integrating quantitative models. Nature Reviews Cancer, 15:730-745, 2015.

[22] A. J. Levine, and M. Oren. The first 30 years of p53: growing ever more complex. Nat. Rev. Cancer, 9:749-758, 2009.

[23] R. A. Gatenby and R. J. Gillies. Why do cancers have high aerobic glycolysis? Nature Reviews Cancer, 4(11):891-899, 2004.

[24] R. A. Gatenby and T. L. Vincent. An evolutionary model of carcinogenesis. Cancer Research, 63(19):6212-6220, 2003.

[25] P. C. W. Davies, L. Demetrius, and J. A. Tuszynski. Cancer as a dynamical phase transition. Theoretical Biology and Medical Modelling, $8(1): 30,2011$.

[26] R. A. Gatenby. Application of competition theory to tumour growth: Implications for tumour biology and treatment. European Journal of Cancer, 32(4):722-726, Apr 1996.

[27] R. Lev Bar-Or, R. Maya, L. A. Segel, U. Alon, A. J. Levine, and M. Oren. Generation of oscillations by the p53-mdm2 feedback loop: A theoretical and experimental study. Proceedings of the National Academy of Sciences, 97(21):11250-11255, 2000.

[28] L. Ma, J. Wagner, J. J. Rice, W. Hu, A. J. Levine, and G. A. Stolovitzky. A plausible model for the digital response of p53 to dna damage. Proceedings of the National Academy of Sciences, 102(40):14266-14271, 2005.

[29] N. Geva-Zatorsky, N. Rosenfeld, S. Itzkovitz, R. Milo, A. Sigal, E. Dekel, T. Yarnitzky, Y. Liron, P. Polak, G. Lahav, and U. Alon. Oscillations and variability in the p53 system. Molecular Systems Biology, 2(1), 2006.

[30] E. Batchelor, C. S. Mock, I. Bhan, A. Loewer, and G. Lahav. Recurrent initiation: A mechanism for triggering p53 pulses in response to dna damage. Molecular Cell, 30(3):277 - 289, 2008.

[31] K. PuszyAski, B. Hat, and T. Lipniacki. Oscillations and bistability in the stochastic model of p53 regulation. Journal of Theoretical Biology, 254(2):452 - 465, 2008.

[32] X. Cai and Z. M. Yuan. Stochastic modeling and simulation of the p53-mdm2/mdmx loop. Journal of Computational Biology, 16(7):917-933, 2009. PMID: 19580521.

[33] X. P. Zhang, F. Liu, and W. Wang. Two-phase dynamics of p53 in the dna damage response. Proceedings of the National Academy of Sciences, 108(22):8990-8995, 2011.

[34] E. Batchelor, A. Loewer, C. Mock, and G. Lahav. Stimulus-dependent dynamics of p53 in single cells. Molecular Systems Biology, $7(1): 488,2011$.

[35] G. B. Leenders and J. A. Tuszynski. Stochastic and deterministic models of cellular p53 regulation. Frontiers in oncology, 3:1229-1244, 2013.

[36] J. Momand, G. P. Zambetti, D. C. Olson, D. George, and A. J. Levine. The mdm-2 oncogene product forms a complex with the p53 protein and inhibits p53-mediated transactivation. Cell, 69(7):1237 - 1245, 1992.

[37] S. Fang, J. P. Jensen, R. L. Ludwig, K. H. Vousden, and A. M. Weissman. Mdm2 is a ring nger-dependent ubiquitin protein ligase for itself and p53. Journal of Biological Chemistry, 275(12):8945-8951, 2000.

[38] R. Honda and H. Yasuda. Activity of mdm2, a ubiquitin ligase, toward p53 or itself is dependent on the ring nger domain of the ligase. Oncogene, 19(11):1473 - 1476, 2000.

[39] J. Roth, M. Dobbelstein, D. A. Freedman, T. Shenk, and A. J. Levine. Nucleo-cytoplasmic shuttling of the hdm2 oncoprotein regulates the levels of the p53 protein via a pathway used by the human immunodeciency virus rev protein. The EMBO Journal, 17(2):554-564, 1998.

[40] Donald W. K. et al. Holland-Frei Cancer Medicine, 6th edition. 2003.

[41] H. Mellert, S. M. Sykes, M. E. Murphy, and S. B. McMahon. The arf/oncogene pathway activates p53 acetylation within the dna binding domain. Cell Cycle, 6(11):1304-1306, 2007. PMID: 17534149.

[42] I. Palmero, M. Murga, A. Zubiaga, and M. Serrano. Activation of arf by oncogenic stress in mouse broblasts is independent of e2f1 and e2f2. Oncogene, 21(19):2939-2947, 2002.

[43] R. Honda and H. Yasuda. Association of p19arf with mdm2 inhibits ubiquitin ligase activity of mdm2 for tumor suppressor p53. The EMBO Journal, 18(1):22-27, 1999.

[44] W. Tao and A. J. Levine. P19arf stabilizes p53 by blocking nucleo-cytoplasmic shuttling of mdm2. Proceedings of the National Academy of Sciences, 96(12):6937-6941, 1999. 
[45] J. D. Weber, L. J. Taylor, M. F. Roussel, C. J. Sherr, and D. Bar-Sagi. Nucleolar arf sequesters mdm2 and activates p53. Nature Cell Biology, 1(1):2026, 1999.

[46] B Roy, J Beamon, E Balint, and D Reisman. Transactivation of the human p53 tumor suppressor gene by c-myc/max contributes to elevated mutant p53 expression in some tumors. Molecular and Cellular Biology, 14(12):7805-7815, 1994.

[47] M. W. Frazier, X. He, J. Wang, Z. Gu, J. L. Cleveland, and G. P. Zambetti. Activation of c-myc gene expression by tumor-derived p53 mutants requires a discrete c-terminal domain. Molecular and Cellular Biology, 18(7):3735-3743, 1998.

[48] M. J. et al. Scian. Tumor-derived p53 mutants induce oncogenesis by transactivating growth-promoting genes. Oncogene, 23(25):4430-4443, 2004.

[49] A. Willis, E. J. Jung, T. Wakeeld, and X. Chen. Mutant p53 exerts a dominant negative eect by preventing wild-type p53 from binding to the promoter of its target genes. Oncogene, 23(13):2330-2338, 2004.

[50] A. de Vries, E. R. Flores, B. Miranda, H. M. Hsieh, C. T. M. van Oostrom, J. Sage, and T. Jacks. Targeted point mutations of p53 lead to dominant-negative inhibition of wild-type p53 function. Proceedings of the National Academy of Sciences, 99(5):2948-2953, 2002.

[51] T. Ozaki and A. Nakagawara. Role of p53 in cell death and human cancers. Cancers, 3(1):994-1013, 2011.

[52] A. Sigal and V. Rotter. Oncogenic mutations of the p53 tumor suppressor: The demons of the guardian of the genome. Cancer Research, 60(24):6788-6793, 2000.

[53] C. Prives and E. White. Does control of mutant p53 by mdm2 complicate cancer therapy? Genes Dev, 22:1259-1264, 2008.

[54] A. Suzuki, R. Kogo, K. Kawahara, M. Sasaki, M. Nishio, T. Maehama, T. Sasaki, K. Mimori, and M. Mori. A new picture of nucleolar stress. Cancer Science, 103(4):632-637, 42012.

[55] B. Roy, J. Beamon, E. Balint, and D. Reisman. Transactivation of the human p53 tumor suppressor gene by c-myc/max contributes to elevated mutant p53 expression in some tumors. Molecular and Cellular Biology, 14(12):7805-7815, 1994.

[56] M. Sachdeva, S. Zhu, F. Wu, H. Wu, V. Walia, S. Kumar, R. Elble, K. Watabe, and Y. Y. Mo. p53 represses c-myc through induction of the tumor suppressor mir-145. Proceedings of the National Academy of Sciences, 106(9):3207-3212, 2009.

[57] J. S. L. Ho, W. Ma, D. Y. L. Mao, and S. Benchimol. p53-dependent transcriptional repression of c-myc is required for g1 cell cycle arrest. Molecular and Cellular Biology, 25(17):7423-7431, 2005.

[58] P. Liao, S. X. Zeng, X. Zhou, T. Chen, F. Zhou, B. Cao, J. H. Jung, G. D. Sal, S. Luo, and H. Lu. Mutant p53 gains its function via c-myc activation upon cdk4 phosphorylation at serine 249 and consequent pin1 binding. Molecular Cell, 68(6):1134-1146.e6, 2017.

[59] G. Saxena, A. Prasad, and R. Ramaswamy. Amplitude death: The emergence of stationarity in coupled nonlinear systems. Physics Reports, 521(5), 205-228, 2012. doi:10.1016/j.physrep.2012.09.003.

[60] Y. Ohiro, I. Garkavtsev, S. Kobayashi, K. R. Sreekumar, R. Nantz, B. T. Higashikubo, S. L. Duy, R. Higashikubo, A. Usheva, D. Gius, N. Kley, and N. Horikoshi. A novel p53-inducible apoptogenic gene, prg3, encodes a homologue of the apoptosis-inducing factor (aif). FEBS Letters, 524(1-3):163-171, 2002.

[61] Q. Luo, J. M. Beaver, Y. Liu, and Z. Zhang. Dynamics of p53: A master decider of cell fate. Genes, 8(2), 2017.

[62] W. H. Homan, S. Biade, J. T. Zilfou, J. Chen, and M. Murphy. Transcriptional repression of the anti-apoptotic surviving gene by wild type p53*. The Journal of Biological Chemistry, 277(5):3247-3257, 2002.

[63] K. Huang, L. Chen, J. Zhang, , and et al. Elevated p53 expression levels correlate with tumor progression and poor prognosis in patients exhibiting esophageal squamous cell carcinoma. Oncol Lett, 8(4):1441-1446, 2014.

[64] P. A. J. Muller, and K. H. Vousden. Mutant p53 in Cancer: New Functions and Therapeutic Opportunities. Cancer Cell, 25: 304-317 (2014)

[65] M. I. Stefan and N. Le Nov'ere. Cooperative binding. PLOS Computational Biology, 9(6):1-6, 062013.

[66] P. Ao, D. Galas, L. Hood, and X. Zhu. Cancer as robust intrinsic state of endogenous molecular-cellular network shaped by evolution. Medical Hypotheses, 70(3):678 - 684, 2008.

[67] T. N. Wong, G. Ramsingh, A. L. Young, C. A. Miller, W. Touma, J. S. Welch, T. L. Lamprecht, D. Shen, J. Hundal, R. S. Fulton, S. Heath, J. D. Baty, J. M. Klco, L. Ding, E. R. Mardis, P. Westervelt, J. F. DiPersio, M. J. Walter, T. A. Graubert, T. J. Ley, T. E. Druley, D. C. Link, and R. K. Wilson. Role of tp53 mutations in the origin and evolution of therapy-related acute myeloid leukaemia. Nature, 518(7540):552-555, 2015.

[68] B. Hat, M. Kochanczyk, M. N. Bogdal, and T. Lipniacki. Feedbacks, bifurcations, and cell fate decision-making in the p53 system. PLoS Comput Biol 12(2):1-28, 02-2016.

[69] R. Mirzayans, B. Andrais, A. Scott, and D. Murray. New insights into p53 signaling and cancer cell response to dna damage: Implications for cancer therapy. Journal of Biomedicine and Biotechnology, 2012: 1-16, 2012.

[70] Y. Shetzer, H. Solomon, G. Koifman, A. Molchadsky, S. Horesh, V. Rotter, The paradigm of mutant p53-expressing cancer stem cells and drug resistance. Carcinogenesis 35(6): 1196-1208, 2014. 
TABLE I: Table 1 Parameters descriptions, and parameter values

\begin{tabular}{|c|c|c|c|c|}
\hline S. No. & Parameter & Value & Description & References \\
\hline 1. & $k_{P}$ & 0.5 proteins $/ \mathrm{s}$ & {$\left[\mathrm{p} 53_{A}\right]$ production } & {$[35]$} \\
\hline 2. & $k_{1}$ & $9.963 \times 10^{-6}$ & {$\left[\mathrm{MDM} 2_{N}\right]$ dependent $\left[\mathrm{p} 53_{A}\right]$ decay } & {$[35]$} \\
\hline 3. & $d_{P}$ & $1.925 \times 10^{-5}$ & {$\left[\mathrm{p} 53_{A}\right]$ decay } & {$[35]$} \\
\hline 4. & $\gamma_{x_{1}}$ & $9.963 \times 10^{-7}$ & {$\left[\mathrm{p} 53_{M}\right]$ dependent $\left[\mathrm{p} 53_{A}\right]$ decay } & Estimated \\
\hline 5. & $\delta_{x_{1}}$ & $4.963 \times 10^{-6}$ & {$\left[\right.$ GENE] dependent $\left[\mathrm{p} 53_{A}\right]$ decay } & Estimated \\
\hline 6. & $K_{1}$ & 50 & Cooperative coefficient & Estimated \\
\hline 7. & $n_{1}$ & 4 & Hill coefficient & Estimated \\
\hline 8. & $\alpha_{x_{2}}$ & $1.5 \times 10^{-7}$ proteins $/ \mathrm{s}$ & {$\left[\mathrm{p} 53_{M}\right]$ production } & Estimated \\
\hline 9. & $\gamma_{x_{2}}$ & $9.963 \times 10^{-6}$ & {$\left[\mathrm{MDM} 2_{N}\right]$ dependent $\left[\mathrm{p} 53_{M}\right]$ decay } & Estimated \\
\hline 10. & $\delta_{x_{2}}$ & $1.925 \times 10^{-5}$ & {$\left[\mathrm{p} 53_{M}\right]$ decay } & Estimated \\
\hline 11. & $\alpha_{x_{3}}$ & $1.5 \times 10^{-7}$ & [GENE] production & Estimated \\
\hline 12. & $\beta_{x_{3}}$ & $5.5 \times 10^{-3}$ & Stress dependent maximum [GENE] activation rate & Estimated \\
\hline 13. & $K_{2}$ & 3 & Cooperative coefficient & Estimated \\
\hline 14. & $n_{2}$ & 3 & Hill coefficient & Estimated \\
\hline 15. & $\delta_{x_{3}}$ & $2.0635 \times 10^{-3}$ & p55 $3_{M}$ dependent maximum [GENE] activation rate & Estimated \\
\hline 16. & $K_{3}$ & 1000 & Cooperative coefficient for oncogene & Estimated \\
\hline 17. & $n_{3}$ & 3 & Hill coefficient & Estimated \\
\hline 18. & $\gamma_{x_{3}}$ & $1.925 \times 10^{-5}$ & [GENE] decay & Estimated \\
\hline 19. & $k_{m}$ & $1.5 \times 10^{-3}$ & {$\left[\mathrm{RNA}_{N}\right]$ production } & {$[35]$} \\
\hline 20. & $k_{2}$ & $1.5 \times 10^{-2}$ & {$\left[\mathrm{p} 53_{A}\right]$ dependent maximum $\left[\mathrm{RNA}_{N}\right]$ activation rate } & {$[35]$} \\
\hline 21. & $k_{D}$ & 740.0 & Cooperative coefficient & {$[35]$} \\
\hline 22. & $k_{0}$ & $8.0 \times 10^{-4}$ & {$\left[\mathrm{RNA}_{N}\right]$ decay and $\left[\mathrm{RNA}_{C}\right]$ production } & {$[35]$} \\
\hline 23. & $d_{r c}$ & $1.444 \times 10^{-4}$ & {$\left[\mathrm{RNA}_{C}\right]$ decay } & {$[35]$} \\
\hline 24. & $k_{T}$ & $1.66 \times 10^{-2}$ & {$\left[\right.$ MDM $\left.2_{C}\right]$ production } & {$[35]$} \\
\hline 25. & $k_{i}$ & $9.0 \times 10^{-4}$ & {$\left[\mathrm{MDM} 2_{C}\right]$ decay and $\left[\mathrm{MDM} 2_{N}\right]$ production } & {$[35]$} \\
\hline 26. & $d_{m n}$ & $1.66 \times 10^{-7}$ & {$\left[\mathrm{MDM} 2_{N}\right]$ decay } & {$[35]$} \\
\hline 27. & $k_{3}$ & $9.963 \times 10^{-6}$ & {$[\mathrm{ARF}]$ dependent $\left[\mathrm{MDM} 2_{N}\right]$ decay } & {$[35]$} \\
\hline 28. & $k_{a}$ & 0.5 proteins $/ \mathrm{s}$ & {$[\mathrm{ARF}]$ production } & {$[35]$} \\
\hline 29. & $K_{4}$ & 10 & Cooperative coefficient for ARF & Estimated \\
\hline 30. & $n_{4}$ & 3 & Hill coefficient & Estimated \\
\hline 31. & $\delta$ & $3.5 \times 10^{-4}$ & {$[\mathrm{ARF}]$ activation rate due to stress } & Estimated \\
\hline 32. & $d_{a}$ & $3.209 \times 10^{-5}$ & {$[\mathrm{ARF}]$ decay } & {$[35]$} \\
\hline 33. & $k_{3}$ & $9.963 \times 10^{-6}$ & {$\left[\mathrm{MDM} 2_{N}\right]$ dependent $[\mathrm{ARF}]$ decay } & {$[35]$} \\
\hline
\end{tabular}

